\title{
The Existences of Positive Solution \\ for Neutral Difference Equations with Muitiply Delay
}

\author{
Department of Mathematics \\ Yanshan University \\ Qinhuangdao 066004, China \\ E-mail: lining0229@163.com
}

Xiaozhu Zhong, Ning Li, Ping Yu, Wenxia Zhang \& Shasha Zhang

Supported by the Foundation for the natural science of Hebei province of China (Z2007431)

\section{Abstract}

We prove the existences of positive solution for neutral difference equation with multiply delay.

Keywords: Difference equation, Positive solution, Much retard

\section{Introduction}

In recent years, Difference equations have beed applied in many areas,such as population dynamics, stability theory, circuit theory, bifurcation analysis, dynamical behavior of delayed network systems and so on. The oscillation or asymptotic behaviour of difference equations was the subject of invesigation by many authors.

In this paper, we are concerned with the following neutral difference equation with positive and negative coefficients.

$$
\Delta\left[x(n)-\sum_{l=1}^{w} R_{l}(n) x\left(n-r_{l}\right)\right]+\sum_{i=1}^{m} P_{i}(n) x\left(n-\tau_{i}\right)-\sum_{j=1}^{k} Q_{j}(n) x\left(n-\sigma_{j}\right)=0\left(^{*}\right)
$$

We will investigate the existences of positive solution of this equation.

where $m \geq k ; R_{l}, P_{i}, Q_{j} \in\left(\left[n_{0}, \infty\right], R^{+}\right) ; r_{l}, \tau_{i}, \sigma_{j}$ are non-negative and non-de-creasing about

$l, i, j, \tau_{i} \geq \sigma_{i}$.

Throughout the paper,we suppose the following assumptions

(1) $H_{i}(n)=P_{i}\left(n+\tau_{i}-\sigma_{i}\right)-Q_{i}(n) \geq 0, n \geq n_{0}, i=1,2, \ldots m$

(2) $H(n)=\sum_{i=1}^{m} H_{i}(n)$

(3) $Q_{\mathrm{j}}(n) \equiv 0, \sigma_{j} \equiv 0, j=k+1, k+2, \ldots m$

When $w=m=k=1$, the equations changed to

$$
\Delta[x(n)-R(n) x(n-r)]+P(n) x(n-\tau)-Q(n) x(n-\sigma)=0 \quad n=0,1, \ldots
$$

Where $\{P(n)\},\{Q(n)\},\{R(n)\}$ are sequence of nonnegative real numbers, $\tau, \sigma, r$ are integers with $0 \leq \sigma \leq \tau-1, r>0$.The oscillatory and non-oscillatory solutions of $\mathrm{Eq}(4)$ have been investigateed by several authors. The aim of the present is to investigate the behavior of eventually positive solutions of $\mathrm{Eq}(1) \mathrm{On}$ the bases of the references of [2].

\section{Some lemmas}

Lemma1 Assume that (1)(2) holds $n \geq n_{0}$ and $\sum_{l=1}^{w} R_{l}(n)+\sum_{i=1}^{m} \sum_{u=n}^{n+\tau_{i}-\sigma_{i}} P_{i}(n) \leq 1$.if $x(n)$ is an eventually positive solutions of the following inequality

$$
\Delta\left[x(n)-\sum_{l=1}^{w} R_{l}(n) x\left(n-r_{l}\right)\right]+\sum_{i=1}^{m} P_{i}(n) x\left(n-\tau_{i}\right)-\sum_{j=1}^{k} Q_{j}(n) x\left(n-\sigma_{j}\right) \leq 0
$$

Setting

$$
y(n)=x(n)-\sum_{l=1}^{w} R_{l}(n) x\left(n-r_{l}\right)+\sum_{i=1}^{m} \sum_{u=n}^{n+\tau_{i}-\sigma_{i}} P_{i}(u) x\left(u-\tau_{i}\right)
$$

then we eventually have $\Delta y(n) \leq 0, y(n)>0$. 
Lemma 2 Assume (1) $\left\{p_{n}\right\}$ are sequences of nonnegative real numbers; (2) $k$ and $l$ are integers; (3) $\left\{q_{n}\right\}$ are sequences of nonnegative real numbers and $p_{n}+l q_{n}>0(n \geq N), l>0$; or $l>0, q_{s} \geq 0 s \in[n . n+1]$

Setting $b=\max \{k, l\}$ assume the inequality

$$
y_{n} \geq p_{n} y_{n-k}+\sum_{s=n}^{\infty} q_{s} \max _{u \in[s-l, s]} y_{u} n \geq N
$$

has an eventually positive solution $\left\{y_{n}\right\}_{N-b}^{\infty}$, then the corresponding difference equation

$$
x_{n}=p_{n} x_{n-k}+\sum_{s=n}^{\infty} q_{s} \max _{u \in[s-l, s]} x_{u} n \geq N
$$

has an eventually positive solution $\left\{x_{n}\right\}_{N-b}^{\infty}$

\section{Main results and proof}

Theorem 1 Assume $\sum_{l=1}^{w} R_{l}(n)+\sum_{i=1}^{m} \sum_{u=n}^{n+\tau_{i}-\sigma_{i}} P_{i}(u) \equiv 1$, equation(*) has an eventually positive solution if and only if the inequality

$$
\Delta\left[x(n)-\sum_{l=1}^{w} R_{l}(n) x\left(n-r_{l}\right)\right]+\sum_{i=1}^{m} P_{i}(n) x\left(n-\tau_{i}\right)-\sum_{j=1}^{k} Q_{j}(n) x\left(n-\sigma_{j}\right) \leq 0
$$

has an eventually positive solution.

Proof: We can see the sufficient condition of the theorem is obvious.we only proof the Necessary condition.

Assume $\{x(n)\}$ is an eventually positive solution of the inequality (5).

define

$$
y(n)=x(n)-\sum_{l=1}^{w} R_{l}(n) x\left(n-r_{l}\right)+\sum_{i=1}^{m} \sum_{u=n}^{n+\tau_{i}-\sigma_{i}} P_{i}(u) x\left(u-\tau_{i}\right)
$$

then in view of lemma 1, we have $\Delta y(n) \leq 0, y(n)>0$. and $\lim _{n \rightarrow \infty} y(n) \geq 0$.

setting $y_{\infty}=\lim _{n \rightarrow \infty} y(n)$ in view of (6), we have

$$
\begin{aligned}
& \Delta y(n)=\Delta\left(x(n)-\sum_{l=1}^{w} R_{l}(n) x\left(n-r_{l}\right)+\sum_{i=1}^{m} \sum_{u=n}^{n+\tau_{i}-\sigma_{i}} P_{i}(u) x\left(u-\tau_{i}\right)\right) \\
& \quad \leq \sum_{j=1}^{k} Q_{j}(n) x\left(n-\sigma_{j}\right)-\sum_{i=1}^{m} P_{i}(n) x\left(n-\tau_{i}\right)-\sum_{i=1}^{m} P_{i}\left(n+\tau_{i}-\sigma_{i}\right) x\left(n-\sigma_{i}\right)+\sum_{i=1}^{m} P_{i}(n) x\left(n-\tau_{i}\right) \\
& \leq \sum_{j=1}^{k} Q_{j}(n) x\left(n-\sigma_{j}\right)-\sum_{i=1}^{m} P_{i}\left(n+\tau_{i}-\sigma_{i}\right) x\left(n-\sigma_{i}\right)=-\sum_{i=1}^{m} H_{i}(n) x\left(n-\sigma_{i}\right) \leq 0
\end{aligned}
$$

Summing both side of the inequality (7) form $n$ to $\infty$ and takeing limt on both side of the resulting inequality, we have

$$
y_{\infty}-y(n) \leq-\sum_{s=n}^{\infty} \sum_{i=1}^{m} H_{i}(s) x\left(s-\sigma_{i}\right)
$$

Then we can see that $\quad y(n) \geq \sum_{s=n}^{\infty} \sum_{i=1}^{m} H_{i}(s) x\left(s-\sigma_{i}\right)+y_{\infty} \geq \sum_{s=n}^{\infty} \sum_{i=1}^{m} H_{i}(s) x\left(s-\sigma_{i}\right)$

$$
\text { i.e } \quad x(n)-\sum_{l=1}^{w} R_{l}(n) x\left(n-r_{l}\right) \geq \sum_{i=1}^{m} \sum_{u=n}^{n+\tau_{i}-\sigma_{i}} P_{i}(u) x\left(u-\tau_{i}\right)+\sum_{s=n}^{\infty} \sum_{i=1}^{m} H_{i}(s) x\left(s-\sigma_{i}\right)
$$

Then the corresponding difference equation

$$
z(n)=\sum_{l=1}^{w} R_{l}(n) z\left(n-r_{l}\right)+\sum_{i=1}^{m} \sum_{u=n}^{n+\tau_{i}-\sigma_{i}} P_{i}(u) z\left(u-\tau_{i}\right)+\sum_{s=n}^{\infty} \sum_{i=1}^{m} H_{i}(s) z\left(s-\sigma_{i}\right)
$$

has an eventually positive solution $z(n)$.obvious $\{z(n)\}$ are positive solutions of $\left(^{*}\right)$.

Theorem 2 Assume $\sum_{l=1}^{w} R_{l}(n)+\sum_{i=1}^{m} \sum_{u=n}^{n+\tau_{i}-\sigma_{i}} P_{i}(u) \equiv 1$.if $\Delta^{2} y(n)+\frac{1}{k} \sum_{i=1}^{m} H_{i}(n) y(n)=0 \quad$ (8)has an eventually positive solution, the the equation $(*)$ aslo has an eventually positive solution.

Proof: Assume $y(n)$ is an eventually positive solution of (8). then we have

$$
\Delta^{2} y(n)=-\frac{1}{k} \sum_{i=1}^{m} H_{i}(n) y(n) \leq 0
$$

Necessarily we have $\Delta y(n)>0$.if it is not true, there must be exist $n_{0}$, if $n \geq n_{0}$, we have $\Delta y(n)<0$, then $y(n)$ exist an nonnegative limt.

Setting $\lim _{n \rightarrow \infty} y(n)=M>0$ 
We have $\Delta^{2} y(n) \leq-\frac{M}{k} \sum_{i=1}^{m} H_{i}(n)$ Summing both side of the inequality form $n_{2}$ to $n$ twice we can see that

$$
y(n+2)-y\left(n_{2}+1\right) \leq-\frac{M}{k} \sum_{s_{2}=n_{2}}^{n} \sum_{s_{1}=s_{2}}^{n} \sum_{i=1}^{m} H_{i}(n)
$$

then $\lim _{n \rightarrow \infty} y(n)=-\infty$ which contradicts with $y(n)$ is an eventually positive solution. so $\Delta y(n)>0$.

we eventually have $y(n)>0, \Delta y(n)>0, \Delta^{2} y(n) \leq 0$.so $\{y(n)\}$ are sequences of nondecreasing, $\{\Delta y(n)\}$ are sequence of nonincreasing setting $a(n)=\Delta y(n)$

we have $a(n)>0, \Delta a(n) \leq 0 n \geq n_{1}$ define a sequence $\{x(n)\}$ as follows

$$
x(n)=\left\{\begin{array}{l}
\frac{y\left(n_{1}\right)}{k}\left(n_{1} \leq n \leq n_{1}+L\right) \\
a(n)+\sum_{l=1}^{w} R_{l}(n) x\left(n-r_{l}\right)+\sum_{i=1}^{m} \sum_{u=n}^{n+\tau_{i}-\sigma_{i}} P_{i}(u) x\left(u-\tau_{i}\right) \\
\left(n_{1}+L+t k \leq n \leq n_{1}+L+(t+1) k\right)(t=0,1,2, \ldots)
\end{array}\right.
$$

then we have $x(n)>0\left(n \geq n_{1}\right)$

$$
\begin{aligned}
& a(n)=x(n)-\sum_{l=1}^{w} R_{l}(n) x\left(n-r_{l}\right)-\sum_{i=1}^{m} \sum_{u=n}^{n+\tau_{i}-\sigma_{i}} P_{i}(u) x\left(u-\tau_{i}\right) n \geq n_{1}+L \\
& x(n)=\frac{y\left(n_{1}\right)}{k} \leq \frac{y(n)}{k}=\frac{1}{k} \sum_{s=n_{1}}^{n-1} a_{s}+\frac{1}{k} y\left(n_{1}\right) \quad n_{1} \leq n \leq n_{1}+L
\end{aligned}
$$

Therefore

$$
\begin{aligned}
x(n)= & a(n)+\sum_{l=1}^{w} R_{l}(n) x\left(n-r_{l}\right)+\sum_{i=1}^{m} \sum_{u=n}^{n+\tau_{i}-\sigma_{i}} P_{i}(u) x\left(u-\tau_{i}\right) \\
\leq & a(n)+\left(\sum_{l=1}^{w} R_{l}(n)+\sum_{i=1}^{m} \sum_{u=n}^{n+\tau_{i}-\sigma_{i}} P_{i}(u)\right)\left(\frac{1}{k} \sum_{s=n_{l}}^{n-k-1} a_{s}+\frac{1}{k} y\left(n_{1}\right)\right) \\
\leq & \frac{1}{k} \sum_{s=n-k}^{n-1} a(s)+\frac{1}{k} \sum_{s=n_{1}}^{n-k-1} a(s)+\frac{1}{k} y\left(n_{1}\right) \\
& =\frac{1}{k} \sum_{s=n_{1}}^{n-1} a(s)+\frac{1}{k} y\left(n_{1}\right)
\end{aligned}
$$

By induction, we can show that

$$
x(n) \leq \frac{1}{k} \sum_{s=n_{1}}^{n-1} a(s)+\frac{1}{k} y\left(n_{1}\right) \quad\left(n_{1}+L+t k \leq n \leq n_{1}+L+(t+1) k\right)(t=0,1,2, \ldots)
$$

and hence

$$
\begin{aligned}
x(n) \leq & \frac{1}{k} \sum_{s=n_{1}}^{n-1} a(s)+\frac{1}{k} y\left(n_{1}\right) \quad n \geq n_{1} \\
x\left(n-\sigma_{i}\right) & \leq \frac{1}{k} \sum_{s=n_{1}}^{n-\tau_{i}-1} a(s)+\frac{1}{k} y\left(n_{1}\right)=\frac{1}{k} y\left(n-\tau_{i}\right) \leq \frac{1}{k} y(n) \quad n \geq n_{1}+L
\end{aligned}
$$

Substituting this into (9) we obtain $\Delta a(n)+\sum_{i=1}^{m} H_{i}(n) x\left(n-\sigma_{i}\right) \leq 0 \quad n \geq n_{1}+L$

So, it follows form (10) that

$$
\begin{gathered}
\Delta\left[x(n)-\sum_{l=1}^{w} R_{l}(n) x\left(n-r_{l}\right)\right]-\sum_{i=1}^{m} P_{i}\left(n+\tau_{i}-\sigma_{i}\right) x\left(n-\sigma_{i}\right)+\sum_{i=1}^{m} P_{i}(n) x\left(n-\tau_{i}\right) \\
+\sum_{i=1}^{m} P_{i}\left(n+\tau_{i}-\sigma_{i}\right) x\left(n-\sigma_{i}\right)-\sum_{i=1}^{m} Q_{i}(n) x\left(n-\sigma_{i}\right) \leq 0
\end{gathered}
$$

i.e

$$
\Delta\left[x(n)-\sum_{l=1}^{w} R_{l}(n) x\left(n-r_{l}\right)\right]+\sum_{i=1}^{m} P_{i}(n) x\left(n-\tau_{i}\right)-\sum_{j=1}^{k} Q_{j}(n) x\left(n-\sigma_{j}\right) \leq 0
$$

By theorem 1, $\mathrm{Eq}(*)$ has an eventually positive solution.

Lemma $3 \operatorname{Let}\{d(n)\}$ be a sequence of nonnegative real numbers,Assume that, for some integer $n^{*}$ and sufficiently large $n$ the inequality 


$$
\left(n-n^{*}\right) \sum_{s=n}^{\infty} d_{s} \leq \frac{1}{4}
$$

holds, Then, the following difference equation

$$
\Delta^{2} y(n)+d_{n} y(n)=0
$$

has an eventually positive solution.

Now we are ready to give out result :

Theorem 3 Assume $\sum_{l=1}^{w} R_{l}(n)+\sum_{i=1}^{m} \sum_{u=n}^{n+\tau_{i}-\sigma_{i}} P_{i}(u) \equiv 1$ holds,for some integer $n^{*}$ and sufficiently large $n$, the inequality $\left(n-n^{*}\right) \sum_{s=n}^{\infty} \sum_{i=1}^{m} H_{i}(s) \leq \frac{k}{4}$

is satisfied, Then, $\mathrm{Eq}\left({ }^{*}\right)$ has an eventually positive positive.

\section{References}

Dong, Weilei. (2006). The oscillation for neutral difference equations with multiply delay. Journal of Hebei Normal University(Natural Science Edition), 30(2): 136-139.

Tang, Xianhua, \& Yu, Jianshe. (2000). Oscillation and nonoscillation of neutral difference equations with positive and negative coefficients. Computer Math Appl, 39: 169-181.

Tian, J.C.(2003). Oscillation Criteria for neutral equations with positive and negative coefficients. Bol SocParana Math, 21: $19-30$.

$\mathrm{Wu}$, Dong. (2003). Oscillation and nonscillation of neutral difference equations with maxima term. JOURNAL OF OCEAN UNIVERSITY OF QINGDAO, 3(6): 975-982.

Zhang, B G, \&Wang, H. (1996). The exience of oscillatory and non-oscillatory solutions of neutral difference equations. Chinese J Math, 24: 377-393. 